\title{
REFLECTION-REFRACTION EFFECTS ON LIGHT DISTRIBUTION INSIDE TUBULAR PHOTOBIOREACTORS
}

\author{
Gaspare Marotta, ${ }^{1}$ Jeremy Pruvost, ${ }^{2}$ Francesca Scargiali, ${ }^{1 *}$ Giuseppe Caputo ${ }^{1}$ and Alberto Brucato ${ }^{1}$ \\ 1. Università di Palermo, Dipartimento dell'Innovazione Industriale e Digitale (DIID), Viale delle Scienze Ed.6, 90128, Palermo, Italy \\ 2. Université de Nantes, CNRS, GEPEA, UMR-CNRS 6144, Bd. De I'Université, CRTT-BP 406, 44620, Saint Nazaire Cedex, France
}

One of the main parameters affecting autotrophic algae cultures is photon absorption distribution inside the photobioreactor. This clearly depends on the geometry of both the radiation source and the photobioreactor, as well as on algae suspension optical properties. In this work the local volumetric rate of photon absorption LVRPA in a cross-section of a horizontal-pipe photobioreactor was investigated by means of simplified Monte Carlo simulations. In particular, the fate of a number of photons perpendicularly hitting the photobioreactor circular section was simulated in relation to different values of algae concentration. The model takes into account refraction/reflection phenomena at the air/photobioreactor-wall interface. Simulation results show that radiation distribution inside the photobioreactor is quite strongly affected by reflection/refraction at the airreactor interface. In particular, dark zones (not revealed when neglecting reflection/refraction phenomena) are observed in conjunction with unexpected radiation intensification in other zones. These phenomena are bound to affect photobioreactor performance and should therefore be considered if effective photobioreactor models are sought.

Keywords: tubular photobioreactor, LVRPA, Monte Carlo, reflection/refraction effects

\section{INTRODUCTION}

$\mathrm{N}$ owadays microalgae play an increasingly important role in the pharmaceutical nutraceutical (for the production of food supplements such as PUFA and carotenoids), cosmetics, and renewable energy (as third-generation bio-fuels) fields.

Taking advantage of photosynthetic processes, microalgae are able to exploit and to convert in an effective way photons, water, $\mathrm{CO}_{2}$, and nutrients into valuable products. ${ }^{[1]}$ In order to obtain high amounts of biomass, microalgae cultures are grown in a variety of photo-bioreactors, including open raceway ponds and closed tubular photobioreactors. For reliable photobioreactor design, suitable models need to be developed. This is particularly hard in this case, as to the biological and hydrodynamic complexities shared with other bioreactors, here also the complexities related to light distribution effects need to be accounted for. As a matter of fact, one of the main parameters affecting autotrophic algae culture performance is photon absorption rate distribution inside the photobioreactor.

The importance of radiative field has been demonstrated in a number of investigations on photosynthetic activity relation with the radiation field involved. ${ }^{[2-5]}$ The knowledge of light distribution inside the photobioreactor is essential especially when photobioreactors are operated at high microalgal cell concentrations. ${ }^{[6]}$ This clearly depends on the geometry of both the radiation source and the photobioreactor, as well as on algae suspension optical properties. Considering a uniform cell distribution, in order to properly model the radiation field inside photobioreactors, the relevant balance equation, known as the Radiation Transfer Equation (RTE), should be solved. This would allow the computation of the local volumetric rate of photon absorption (LVRPA), a quantity strictly related to photosynthetic growth rate and biomass generation. ${ }^{[7-9]}$

Considering that the integro-differential nature of RTE makes it difficult to obtain even numerical solutions, alternative methods have been developed. For instance, Colina et al. ${ }^{[10]}$ used a P1 approach for solving the RTE in solar tubular photoreactors and Machuca et al. ${ }^{[11]}$ used an hybrid approach by fitting model parameters to experimental data. Another interesting approach is the use of Monte Carlo methods, which allow rigorous numerical solutions to be obtained in a much simpler way than solving the full RTE ${ }^{[12]}$ Fundamentals of this technique are well-known. ${ }^{[13]}$ For instance, Brucato et al. ${ }^{[14]}$ employed it for validating the simplified "Six Flux" radiation model in slab photo-catalytic reactors while Busciglio et al. ${ }^{[15]}$ employed it to model radiation transfer in "quasi-isoactinic" reactors.

Taking into account this last approach, the purpose of this work is that of studying the LVRPA distribution over a cross-section of a horizontal-pipe photobioreactor. In particular, a Monte Carlo method was employed to simulate a photobioreactor irradiated by a far-away external radiation source. Tubular closed photobioreactors are commonly used for solar cultures, together with flat panel PBRs. However light distribution simulation is not straightforward in tubular geometries due to complexities related to refraction effects on curved walls. As a difference, flat panel PBRs which respond to the so-called "one-dimensional approximation" are much more amenable to simple simulations. ${ }^{[16,17]}$

\section{METHODOLOGY}

\section{Mathematical Modelling}

The investigated geometry is a tubular photobioreactor with internal radius $R$ and parallel irradiation from the outside, as it 
would occur with direct sunlight or any other relatively far away source. In Figure 1 a schematic diagram of the crosssection is reported. It is worth noting that the investigated condition is the specific one encountered at noon for an eastwest oriented tube. Also in this case, the irradiation is bound to have a time varying incident angle with respect to solar course. Though the case here investigated may be regarded as a quite singular condition, it was considered sufficiently informative on the peculiar phenomena that take place in these systems. Other tilt angle effects will be the subject of subsequent work.

The tubular reactor simplified mathematical modelling is based on the assumption that photon path is reflected and refracted, according to Fresnel and Snell laws, when passing from one medium to another with a different refraction index, as depicted in Figure 1. For the sake of simplicity, the deflection effects due to the thin transparent wall of the tubular photobioreactor were neglected, as suggested by Pruvost et al. ${ }^{[16]}$ and Lee et al. ${ }^{[17]}$ The history of a statistically meaningful number of photons, $N_{r}$, travelling inside a generic photobioreactor cross-section was simulated.

It is worth noting that the absorption and scattering properties of the medium affect the mean free path of the photons travelling inside the photobioreactor. Considering the microscopic size of microalgae, these can be modelled as uniformly distributed particles in the culture broth. Therefore, in relation to different values of microalgae concentration, $C_{x}$, the fate of a large number of photons was simulated, starting from its entrance into the photobioreactor cross-section until they either escaped from the photobioreactor or were absorbed inside the growth medium. A simplified approach was also used as concerns photon-algae interactions, namely scattering and diffraction effects were neglected and all interactions were assumed to result in photon absorption by the microalgae. This assumption was made in view of the strongly forward oriented scattering phase function experimentally measured by Kandilian et al., ${ }^{[18]}$ that results in very small deflection effects when a scattering event takes place, so making acceptable the assumption of no deflection upon

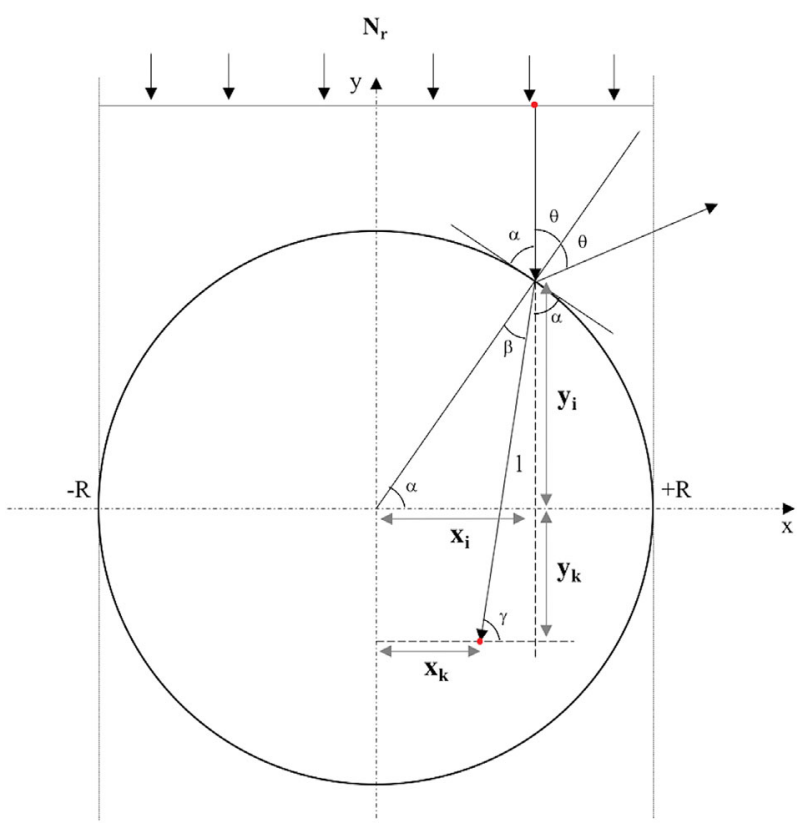

Figure 1. Irradiated cross-section by photons taking into account Fresnel and Snell laws. scattering. Finally, reflection effects at the inner medium/air interface were also neglected, in view of their negligible extent at sufficient microalgae concentrations. ${ }^{[19]}$

The procedure was started by randomly selecting the photon offset with respect to tube axis:

$x_{i}=R_{n 1} \cdot R$

where $R_{n 1}$ is a random number between -1 and 1 .

Then accordingly, the ordinate is simply derived by the circle equation as:

$y_{i}=\sqrt{\left(R^{2}-x_{i}^{2}\right)}$

According to Snell's law, which quantifies refraction at the air/wall interface, the deviation angle $\beta$ of the photon into the photobioreactor is given by:

$\beta=\sin ^{-1}\left(\sin \theta \cdot \frac{n_{1}}{n_{2}}\right)$

where $\theta$ is the angle between the direction of photon travel and the normal direction and $n_{1}$ and $n_{2}$ are the refraction indexes of air (1.0) and water (1.33) respectively. As previously stated, it is considered that the photobioreactor wall is so thin as to not to affect the optical diffraction phenomena.

Once a photon has entered the photobioreactor space, while travelling it is subject to hitting microalgae cells. As the probability of hitting a cell is uniform, an exponential decay of radiation intensity can be presumed, which is characterized by a characteristic extinction length, $\lambda$. This last is inversely proportional to the product of microalgae concentration, $C_{x}$, by the radiant energy mass coefficient, $E_{a}:{ }^{[7]}$

$\lambda=\frac{1}{\mathrm{C}_{\mathrm{x}} \mathrm{E}_{\mathrm{a}}}$

As a consequence, the distance travelled by the photon before hitting a cell and being absorbed was computed as:

$l_{i}=-\lambda \ln \left(R_{n 2}\right)$

where $\mathrm{R}_{n 2}$ is a new random number between zero and one. The coordinates $\left(x_{k}, y_{k}\right)$ where the absorption event occurs are therefore given by, this depth is given by:

$x_{k}=x_{i}-l_{i} \cos \gamma=x_{i}-\lambda \ln \left(R_{n 2}\right) \cos \gamma$

$y_{k}=y_{i}-l_{i} \sin \gamma=x_{i}-\lambda \ln \left(R_{n 2}\right) \sin \gamma$

where $\gamma$ is given by the sum of angles $\alpha=\pi / 2-\theta$ and $\beta$, as shown in Figure 1.

Obviously, when the condition $\left(x_{k}^{2}+y_{k}^{2}>R^{2}\right)$ is found to be true, then this implies that the photon has exited the reactor space without being affected by absorption phenomena. In the other case, photon absorption has occurred inside the reactor space and the relevant coordinates are recorded for subsequent processing.

As is well known, at the air/medium interface the refracted fraction of energy is smaller than 1, due to the loss of reflected radiant energy. This might have been accounted for by generating a further random number and deciding whether 
the photon has been reflected or refracted according to the relevant probabilities. The choice here adopted was instead that of assuming that the refracted photon carries a "weight" smaller than 1, and equal to the refracted fraction of energy. This is akin to following the fate of a number of photons, belonging to the same parcel and assuming they all undergo the same absorption events.

In order to compute the refracted energy fraction, if the incident photons are not polarized and medium culture is assumed to be non-magnetic, the Fresnel laws for a)

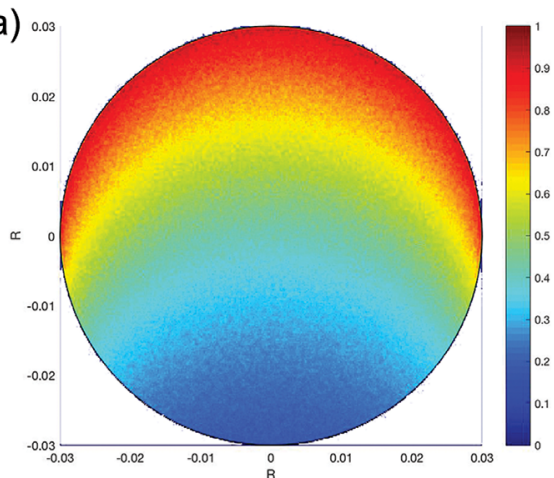

c) 0.0

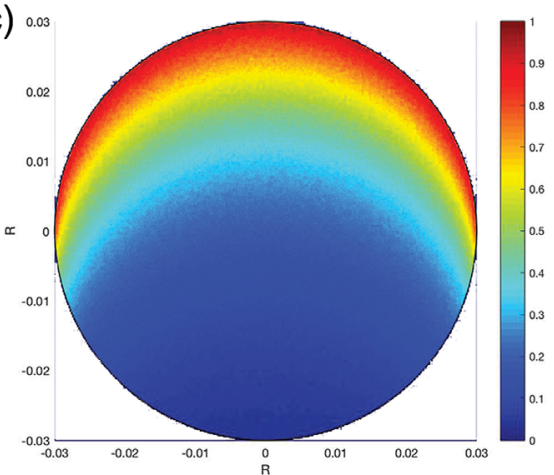

e)

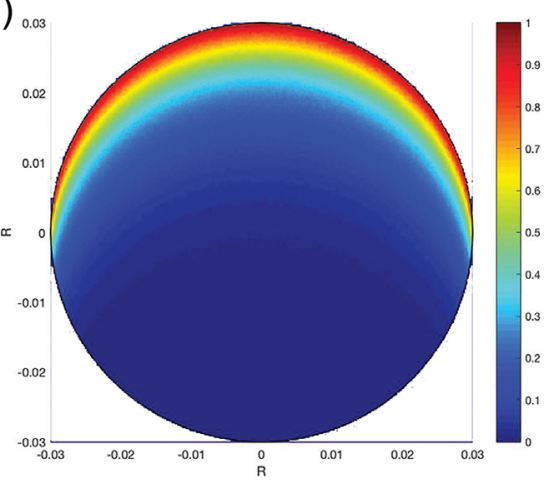

g) 0.00

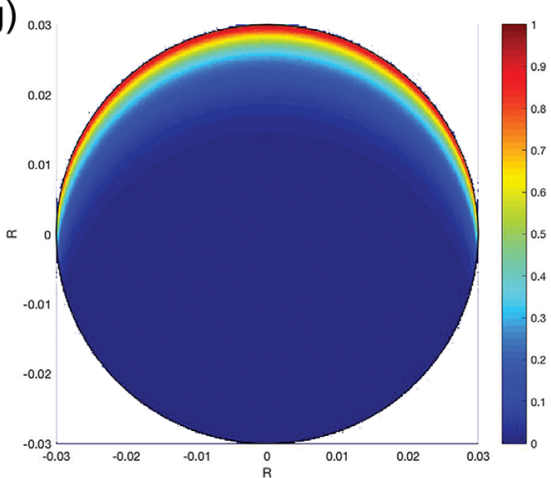

b) 0.03

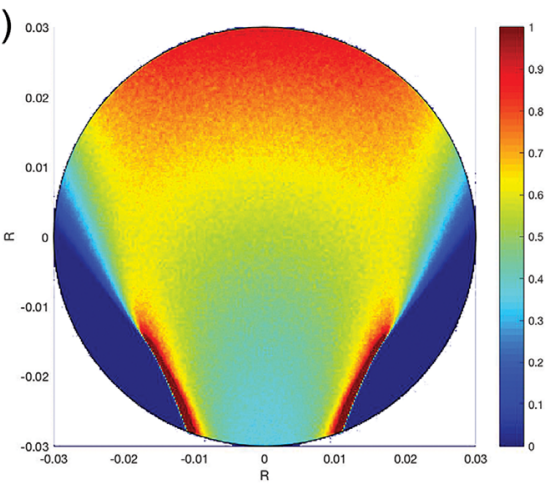

d) 0.00

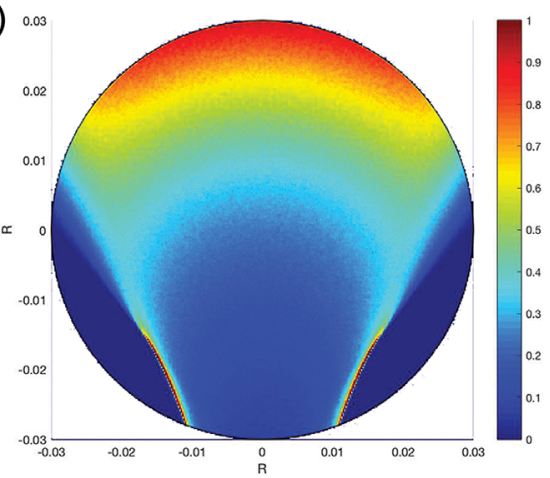

f) 0.0

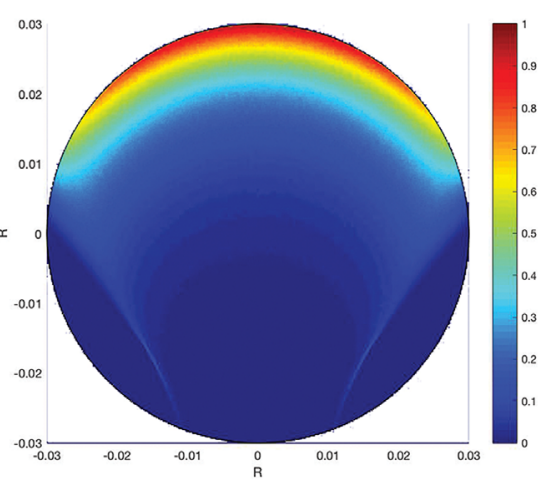

h)

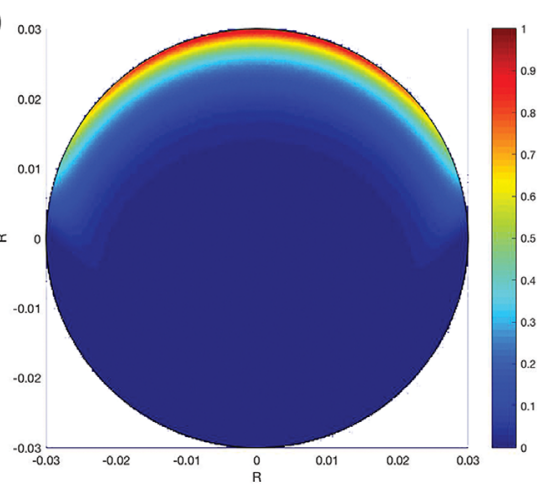

Figure 2. Contour maps of normalized LVRPA at different biomass concentration: (a-b) $C_{x} 0.10 \mathrm{~kg} / \mathrm{m}^{3} ;(\mathrm{c}-\mathrm{d}) C_{x} 0.2 \mathrm{~kg} / \mathrm{m}^{3} ;(\mathrm{e}-\mathrm{f}) C_{x} 0.5 \mathrm{~kg} / \mathrm{m}^{3}$; (g-h) $C_{x}$ $1.0 \mathrm{~kg} / \mathrm{m}^{3}$ : left-side $(a, c, e, g)$ refraction/reflection not considered, right-side $(b, d, f, h)$ refraction/reflection at reactor wall included in the model. 
electromagnetic energy conservation can be employed. According to these, the reflected energy fraction is given by:

$R_{f}=\frac{R_{s}+R_{p}}{2}=\frac{1}{2}\left\{\left[\frac{\left(n_{1} \cos \theta-n_{2} \cos \beta\right)}{\left(n_{1} \cos \theta+n_{2} \cos \beta\right)}\right]^{2}+\left[\frac{\left(n_{1} \cos \beta-n_{2} \cos \theta\right)}{\left(n_{1} \cos \beta+n_{2} \cos \theta\right)}\right]^{2}\right\}$

where $R_{s}$ and $R_{p}$ are the reflectance for s-polarized light and for p-polarized light respectively.

Therefore, the transmitted energy fraction is computed as:

$T=1-R_{f}$

Each photon entering the reactor space is therefore assumed to carry a weight equal to $T$, and when absorbed inside the reactor, its weight is recorded along with its absorption site coordinates.

A typical computation involved following the fate of a large number of photon parcels, resulting in a smaller number of absorption events inside the reactor space, each one characterized by its coordinates and weight.

\section{LVRPA Distribution Assessment}

In order to obtain the LVRPA distribution, the cross-section of the photobioreactor was discretized with a $2 \mathrm{D}$ structured mesh of $300 \times 300(90000)$ square elements. For each of the photon parcels simulated, the mesh element in which absorption had taken place was identified and the parcel weight was added to the relevant element of a square matrix. The result was finally normalized by dividing it by the total number of photon parcels simulated and multiplying the result by the number of square cells. In this way in each element of the matrix a number proportional to the local rate of photon absorption was obtained.

By exploring several total numbers of photon parcels, it was found that, with the spatial resolution adopted $(300 \times 300)$, a number of 50000000 photons was more than sufficient to obtain stable and smooth results.

By using MATLAB as the development environment, even in the most demanding cases, CPU times never exceeded a few tens of seconds on the common PC employed for the calculations.

a)

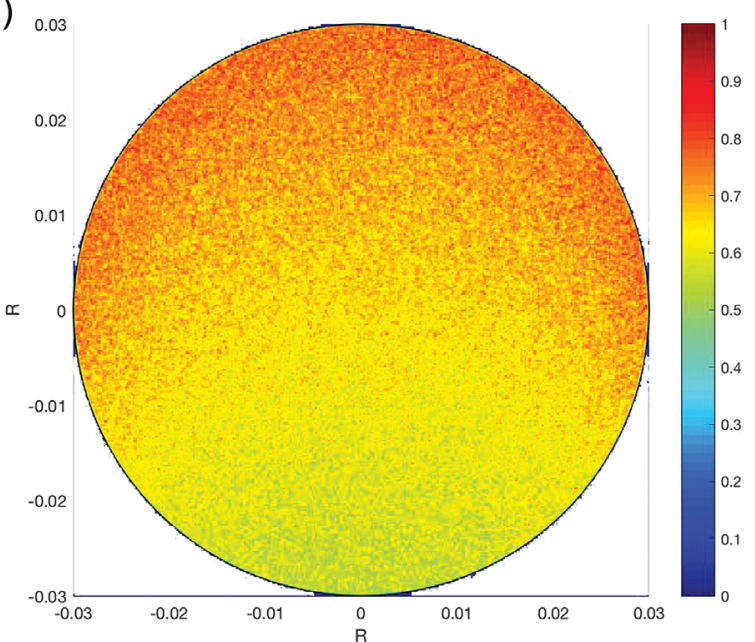

\section{RESULTS AND DISCUSSION}

All results are reported as normalized LVRPA for convenience of analysis.

LVRPA distributions were obtained for different values of the characteristic extinction length $\lambda$ and then, for different biomass concentrations with fixed value of $E_{a}$ by means of the developed Monte Carlo code. The results obtained can be compared with the corresponding LVRPA distributions obtained by neglecting the optical refraction/reflection phenomena at the air liquid interface, as it is customarily made in the relevant open literature. ${ }^{[17,20]}$ Such a comparison is shown in Figure 2, where the results obtained in relation to four different values of algae biomass concentration $C_{x}$ $\left(0.10,0.20,0.5,1.0 \mathrm{~kg} / \mathrm{m}^{3}\right)$ are reported. In particular, on the left side the results obtained while neglecting reflection/refraction phenomena are shown (Figures 2a, c, e, g) and as can be seen, the distributions obtained are consistent with similar results reported in the open literature. Please note that with the aim of increasing the map readability, each map is normalized by dividing all values by the maximum LVRPA value observed in the same map. As can be observed, the smaller the biomass concentration the more spread-out the LVRPA distribution is (though with smaller absolute values at all points, a feature not revealed by the normalized maps), as could have been expected.

On the right side of Figure 2 the results obtained at the same concentrations are shown, but this time by considering the reflection/refraction phenomena at the photobioreactor wall. Reported values are normalized by the same maximum value as the relevant figure at the left. As can be seen the LVRPA distribution is quite strongly affected by reflection/refraction phenomena. In particular, by comparing Figures $2 \mathrm{a}$ and $2 \mathrm{~b}$, both obtained at the same biomass concentration of $0.10 \mathrm{~kg} / \mathrm{m}^{3}$, the existence of completely dark zones in the lower side areas in Figure $2 b$, not present in Figure 2a, can be observed. This clearly depends on the fact that due to refraction angles directing photons towards the reactor centre, those photobioreactor zones are never reached by any photon. This is a feature not revealed by the simpler, but less realistic, simulation of Figure $2 \mathrm{a}$. Due to the sensitivity of algae productivity to passages into dark zones, this may well be an important feature to spot, if accurate simulations of photobioreactor performance are sought.

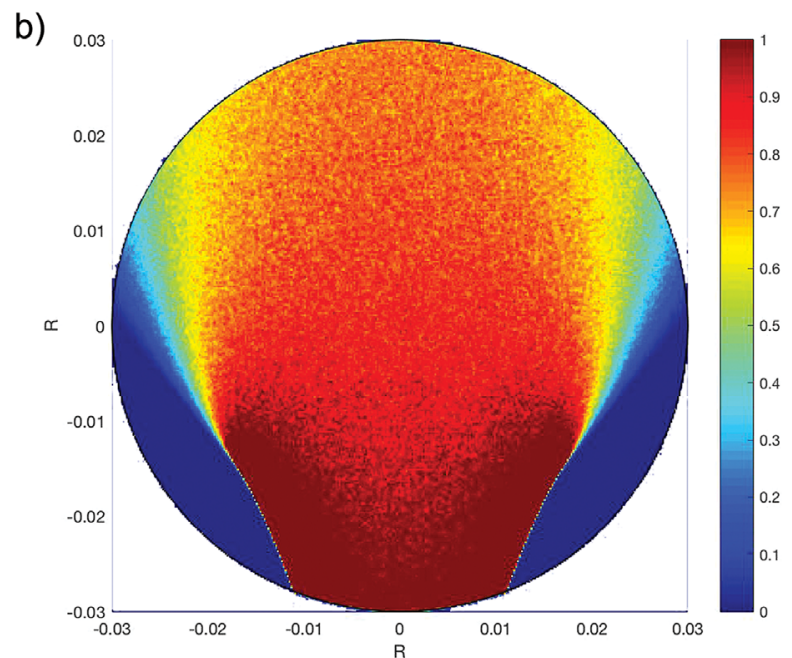

Figure 3. Contour maps of normalized LVRPA at low biomass concentration $\left(C_{x} 0.02 \mathrm{~kg} / \mathrm{m}^{3}\right)$ : (a) refraction/reflection not considered, (b) refraction/reflection at reactor wall included in the model. 
Table 1. Percentage of incident photons lost (not absorbed inside the photobioreactor) at various algae concentrations

\begin{tabular}{lcccll}
\hline & \multicolumn{5}{c}{ Photon loss (\%) } \\
\hline$C_{x}(\mathrm{~g} / \mathrm{L})$ & 0.02 & 0.1 & 0.2 & 0.5 & 1.0 \\
Refraction/reflection considered & 75.85 & 26.96 & 9.89 & 4.56 & 4.46 \\
$\begin{array}{l}\text { Refraction/reflection not } \\
\quad \text { considered }\end{array}$ & 77.73 & 30.19 & 10.93 & 1.62 & 0.39 \\
\hline
\end{tabular}

Notably, in Figure $2 \mathrm{~b}$ a strong LVRPA concentration in the close vicinity of the dark zones is also observed, once again due to the photon redirection effects at the photobioreactor walls.

By comparing Figures 2d, f, h with their left side mates, the already described effects are observed, though their importance becomes progressively less important, due to the smaller and smaller photon penetration depths.

On the contrary, going to much smaller microalgae concentrations, such as at $0.02 \mathrm{~kg} / \mathrm{m}^{3}$, the same effects are further enhanced, as can be appreciated in Figures 3a and $3 \mathrm{~b}$. A striking feature observable in Figure $3 b$ is that the largest LVRPA values are observed in the bottom part of the photobioreactor, in the farthest zones from light entrance, due to the focusing lens effect involved in photon redirection by refraction. Obviously, this feature is not captured when neglecting refraction/reflection phenomena, so resulting in Figure 3a, where a less realistic, much more uniform, LVRPA distribution is predicted.

As regards the number of photons lost in the various conditions by both transmission and reflection, this is reported in Table 1. As can be seen, by increasing biomass levels these percentages decrease, implying an increased number of absorbed photons into the photobioreactor. Notably at high algae concentrations the predicted photon percent loss is higher when considering reflection/refraction phenomena, due to the photons reflected at the air/liquid interface, not accounted for when neglecting reflection. On the contrary, at low algae concentrations the predicted photon percent loss is found to be smaller when considering reflection/refraction phenomena than when neglecting them. This is due to the significantly longer path inside the reactor for many refracted photons, especially those entering the reactor at large $x$ values (see Figure 1), which makes for a larger absorption probability, so offsetting the already quoted reflection effect.

This is also clearly observable in Figure 4, where fractional photon absorption is plotted versus microalgae concentration, where it can be seen that the two effects exactly compensate at algae concentration of about $0.20 \mathrm{~kg} / \mathrm{m}^{3}$, while compensation is only partial at all other concentrations. Overall one can state that, due to the partial compensation of increased photon loss due to reflection and decreased photon loss due to longer escape paths, either considering or neglecting the reflection/refraction phenomena has a minor effect on the percent photon absorption inside the reactor. The major effect remains therefore that on the LVRPA distribution, which may be strikingly different in the two cases, especially at relatively low algae concentrations. This effect is especially bound to play a significant role in the resulting photosynthetic growth, as light distribution and especially dark volumes are known to significantly affect resulting light conversion by microalgae and then photobioreactor performances.

It is worth noting that exactly the same LVRPA distribution (hence the same percent photon loss) is obtained for equal values of the dimensionless ratio $R / \lambda$. As for given algae optical properties and for relatively dilute systems, namely for algae volume occupation not exceeding $0.5 \%$ (i.e. $5 \mathrm{~g} / \mathrm{L}$ ), $\lambda$ is simply inversely proportional to algae volume fraction $\phi:^{[12]}$

$\lambda={ }^{2} / 3 \frac{d_{32}}{\phi}$

and therefore to algae concentration $C_{x}$, it clearly stems that the parameter that fully defines the radiant field distribution (and related quantities, such as LVRPA distribution and fractional photon losses) is in practice the product $\left(C_{x} \cdot R\right)$. This consideration implies that if $0.5 \mathrm{~g} / \mathrm{L}$ is needed with a $0.03 \mathrm{~m}$ radius PBR to reduce photon loss to $4.56 \%$ (see Table 1 ) then

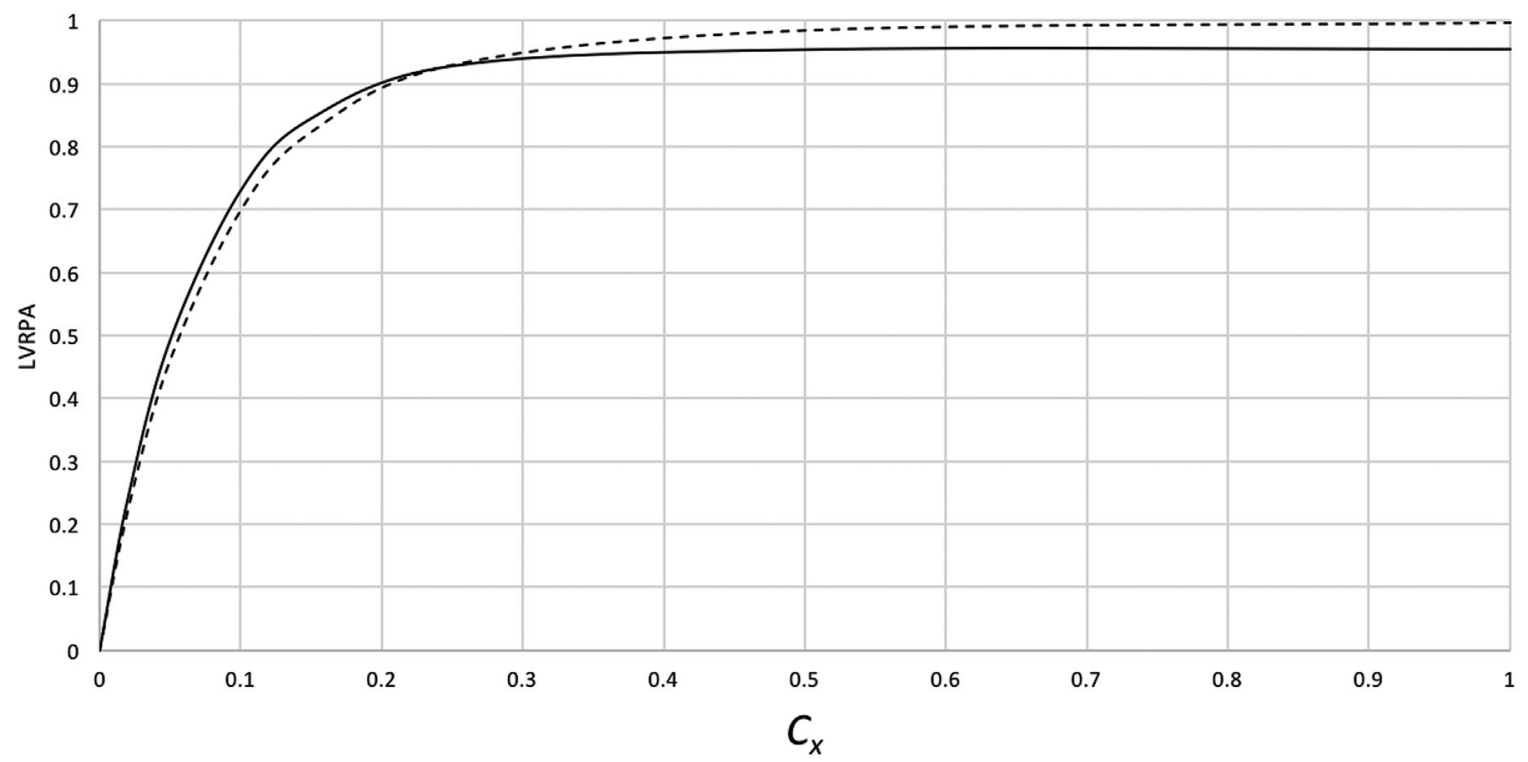

Figure 4. Fraction of incident photons absorbed inside the photobioreactor versus microalgae biomass concentration, with refraction/reflection at reactor wall considered (solid line) or not (dotted line). 
in a $0.06 \mathrm{~m}$ radius PBR $0.25 \mathrm{~g} / \mathrm{L}$ will provide exactly the same result (same distribution as in Figure $2 \mathrm{f}$ and same percent photon loss of 4.56 ).

\section{CONCLUSIONS}

The local volumetric rate of photon absorption (LVRPA) distribution over the cross-section of a horizontal-pipe photobioreactor was determined. A uniform orthogonal irradiation was considered for all the cases analyzed. A simplified mathematical model based on the Monte Carlo approach was adopted, in order to evaluate the influence on LVRPA of implementing optical reflection/diffraction at photobioreactor walls. Results confirm that tubular photobioreactor is submitted to a strongly heterogeneous light attenuation field as induced by wall curvature, inducing in the culture volume both areas of high LVRPA and dark volumes. The overall photon absorption is only marginally affected by refraction/reflection phenomena, while the same phenomena may quite strongly affect the LVRPA distribution inside the reactor, especially at low algae concentrations. As a consequence, refraction/reflection effects may be neglected in strongly simplified models involving only the average LVRPA while the same effects should be considered in advanced photobioreactor models aimed at predicting photosynthetic conversion, as in such cases LVRPA values and especially dark volumes are bound to significantly affect model results.

\section{NOMENCLATURE}

RTE Radiation Transfer Equation

LVRPA Local Volumetric Rate of Photon Absorption (Einstein/ $\mathrm{m}^{3} / \mathrm{s}$ )

$R \quad$ tubular photobioreactor radius (m)

$\mathrm{Nr} \quad$ statistically meaningful number of photons

$C_{x} \quad$ microalgae concentration $\left(\mathrm{kg} / \mathrm{m}^{3}\right)$

$\lambda \quad$ characteristic extinction length (m)

$E_{a} \quad$ radiant energy absorption coefficient $\left(\mathrm{m}^{2} / \mathrm{kg}\right)$

$R_{n i} \quad$ random numbers

$l_{i} \quad$ length travelled by photons before being absorbed (m)

$\alpha \quad$ angle between the direction of photon travel and normal direction (rad)

$\beta \quad$ deviation angle of the photon (rad)

$\theta \quad$ angle between the direction of photon travel and normal direction (rad)

refraction indexes

sum of angles $\alpha$ and $\beta$ (rad)

transmittance (Einstein $/ \mathrm{m}^{2} / \mathrm{s}$ )

reflectance (Einstein $/ \mathrm{m}^{2} / \mathrm{s}$ )

reflectance for s-polarized light

reflectance for p-polarized light (Einstein $/ \mathrm{m}^{2} / \mathrm{s}$ )

\section{REFERENCES}

[1] A. Richmond, Q. Hu, Handbook of Microalgal Culture, 2nd edition, John Wiley \& Sons, Hoboken 2013, p. 102.

[2] A. K. Pegallapati, Y. Arudchelvam, N. Nirmalakhandan, Bioresource Technol. 2012, 126, 266.

[3] S. Xue, Q. Zhang, X. Wu, C. Yan, W. Cong, Bioresource Technol. 2013, 138, 141.

[4] M. Janssen, Cultivation of microalgae: effect of light/dark cycles on biomass yield, PhD thesis, Wageningen University, Wageningen 2002, 184.
[5] R. Pandey, A. Sahu, K. K. Vasumathi, M. Premalatha, Bioproc. Biosyst. Eng. 2015, 38, 1547.

[6] B. Kong, R. D. Vigil, Bioresource Technol. 2014, 158, 141.

[7] A. Souliès, J. Legrand, H. Marec, J. Pruvost, C. Castelain, T. Burghelea, J. F. Cornet, Biotechnol. Progr. 2016, 1, 1.

[8] J. Pruvost, F. Le Borgne, A. Artu, J. Legrand, Algal Research 2017, 21, 158.

[9] R. Kandilian, J. Pruvost, J. Legrand, L. Pilon, Bioresource Technol. 2014, 163, 124.

[10] J. Colina, F. Machuca-Martínez, G. Li Puma, Environ. Sci. Technol. 2010, 44, 5112.

[11] F. Machuca-Martínez, J. Colina-Marquez, M. Mueses, J. Adv. Oxid. Technol. 2008, 11, 1.

[12] A. Brucato, A. E. Cassano, F. Grisafi, G. Montante, L. Rizzuti, G. Vella, AIChE J. 2006, 52, 11.

[13] D. C. Hammersley, J. M. Handscomb, Monte Carlo Methods, 1st edition, Chapman and Hall, London 1983, 34.

[14] A. Brucato, F. Grisafi, L. Rizzuti, A. Sclafani, G. Vella, Ind. Eng. Chem. Res. 2007, 46, 7684.

[15] A. Busciglio, O. M. Alfano, F. Scargiali, A. Brucato, Chem. Eng. Sci. 2016, 142, 79.

[16] J. Pruvost, J. F. Cornet, F. Le Borgne, V. Goetz, J. Legrand, Algal Research 2015, 10, 1.

[17] E. Lee, J. Pruvost, X. He, R. Munipalli, L. Pilon, Chem. Eng. Sci. 2014, 106, 18.

[18] R. Kandilian, J. Pruvost, A. Artu, C. Lemasson, J. Legrand, L. Pilon, J. Quant. Spectrosc. Ra. 2016, 175, 30.

[19] R. Kandilian, A. Soulies, J. Pruvost, B. Rousseau, J. Legrand, L. Pilon, Chem. Eng. Sci. 2016, 146, 1.

[20] E. Molina, F. Fernández, J. Appl. Phys. 2000, 12, 355. 